\title{
Microparticles: A Novel Approach to Enhance Drug Bioavailability
}

\section{Yacobucci MJ and Chablani $\mathrm{L}^{*}$}

Wegmans School of Pharmacy, St. John Fisher College, Rochester, NY 14618, USA

This editorial article reviews the use of microparticles as a drug delivery system to enhance bioavailability of active pharmaceutical ingredients. Bioavailability is defined as the rate and extent of drug absorption from a dosage form into the systemic circulation. Poor bioavailability of drug molecules has been a consistent concern for the pharmaceutical industry and several techniques have been explored to address this issue. There are several factors that can lead to poor bioavailability of a drug molecule:
a. Drug dissolution
b. Drug instability
c. Permeability through epithelial membrane
i. Low partition coefficient
ii. Unsuitable $\mathrm{pKa}$
iii. Large molecular size
d. Pre-systemic metabolism
e. Drug interactions
f. Physiologic issues pertaining to the patient

Microparticulate drug delivery systems have been studied by the researchers for several years for various applications including targeted drug delivery, reduced drug toxicity and enhanced bioavailability of drug molecules. These microparticles can range from 1-200 $\mu \mathrm{m}$ for most of these applications. These micron sized drug delivery systems can be prepared using different techniques, such as, spray drying, emulsion method, jet milling, adsorption and solvent evaporation, fluidization/ solvent precipitation, phase separation and interfacial polymerization methods. Literature validates that various polymers have been used to prepare microparticles using these techniques, ranging from natural polymers (albumin, gelatin, chitosan, starch, amylodextrins, cellulose derivatives etc.) to synthetic polymers (poly glycolic acid- PLGA, poly vinyl alcohol-PVA etc.) based on the study requirements.

Several research studies have reported the use of microparticles to enhance oral bioavailability of drug molecules. Zhang et al. described the use of carboxylated mesoporous carbon microparticles (cMCMs) to increase the oral bioavailability of carvedilol, a poorly water soluble beta-blocker [1]. Here, the cMCMs were prepared by adsorption and solvent evaporation technique. These rod-like particles were 1-2 $\mu \mathrm{m}$ long and $0.5 \mu \mathrm{m}$ in diameter. The study concluded that the use of the cMCMs can enhance the bioavailability of carvedilol by $179.3 \%$ compared to the commercial product.

Similarly, Seremeta et al. studied the use of spray dried poly epsiloncaprolactone (PCL) microparticles containing didanosine, a nucleoside reverse transcriptase inhibitor (NRTI) antiretroviral drug [2]. The average diameter of these spherical microparticles was around 36-118 micrometers and they were tested in vitro and in vivo to determine the oral bioavailability of the drug. The study reports a 2.5 -fold increase in oral bioavailability of didanosine compared to the free drug solution when evaluated in a rat model.

Mebendazole is another poorly absorbed oral drug due to its hydrophobic nature, accounting for its clinically variable results. De la Torre-Iglesias et al. prepared redispersible microparticles containing mebendazole in a 1:2.5 and 1:5 ratio of mebendazole: L-HPC close parenthesis after hydroxypropylcellulose [3]. They observed a 2.67 and 2.97-fold increase in bioavailability for redispersible microparticles containing mebendazole prepared at 1:2.5 and 1:5 ratios of mebendazole: L-HPC, respectively. Redispersible microparticles containing mebendazole at a 1:5 ratio of mebendazole: L-HPC were most effective in reducing the number of Trichinella spiralis parasites (4.56-fold increase) as compared to pure mebendazole at the encysted stage.

Another example for this application is metformin. The low bioavailability of metformin can be attributed to its shortened retention time in the gastrointestinal tract and is enhanced when gastrointestinal motility is slowed. Khonsari et al. prepared gastric-mucoadhesive disk microparticles of metformin by emulsification solvent evaporation using different ratios of carbomer 934p and ethylcellulose [4]. Their mucoadhesive disk microparticles effectively prolonged the release of metformin by 8 hours (71.65-82.22\%). These results are significant and can be very useful if clinically translated.

Further, Al Kindi et al. prepared milrinone-PLGA loaded microparticles by double emulsion-solvent evaporation and evaluated them in a rodent model [5]. The in vivo results demonstrated that the drug plasma levels, taken 24 hours after injection, were significantly higher in mice receiving milrinone-PLGA loaded microparticles (9.1 $\pm 2.2 \mathrm{ng} / \mathrm{mL})$ as compared to milrinone alone $(1.7 \pm 2.4 \mathrm{ng} / \mathrm{mL})[(\mathrm{p}$ $<0.05)]$.

Thus, the literature confirms the use of microparticles as a drug delivery system to enhance oral bioavailability of drug molecules. As there are various methods and polymers available to prepare these microparticles, it provides enormous opportunity to the pharmaceutical scientists to invest in this technology and address bioavailability issues of drugs from various classifications. There are several equipment available to produce these microparticles for commercial purposes (spray dryers and lyophilizers), making them lucrative to be scaled up and meet the needs of the patients.

\section{References}

1. Zhang Y, Zhi Z, Li X, Gao J, Song Y (2013) Carboxylated mesoporous carbon microparticles as new approach to improve the oral bioavailability of poorly water-soluble carvedilol. Int J Pharm 454: 403-411.

2. Seremeta KP, Tur MI, Perez SM, Hocht C, Taira C, et al. (2014) Spray-dried

*Corresponding author: Chablani L, Department of Pharmaceutical Sciences, Wegmans School of Pharmacy, St. John Fisher College, Rochester, NY 14618, USA Tel: +1-585-899-3714; Email: Ichablani@sjfc.edu

Received May 29, 2015; Accepted May 30, 2015; Published June 06, 2015

Citation: Yacobucci MJ, Chablani L (2015) Microparticles: A Novel Approach to Enhance Drug Bioavailability. J Bioequiv Availab 7: e68. doi:10.4172/jbb.10000e68

Copyright: (c) 2015 Yacobucci MJ, et al. This is an open-access article distributed under the terms of the Creative Commons Attribution License, which permits unrestricted use, distribution, and reproduction in any medium, provided the original author and source are credited. 
Citation: Yacobucci MJ, Chablani L (2015) Microparticles: A Novel Approach to Enhance Drug Bioavailability. J Bioequiv Availab 7: e68. doi:10.4172/ jbb.10000e68

Page 2 of 2

didanosine-loaded polymeric particles for enhanced oral bioavailability. Colloids Surf B Biointerfaces 123: 515-523.

3. De La Torre-Iglesias PM, Garcia-Rodriguez JJ, Torrado G, Torrado S, Torrado-Santiago S, et al. (2014) Enhanced bioavailability and anthelmintic efficacy of mebendazole in redispersible microparticles with low-substituted hydroxypropylcellulose. Drug Des Devel Ther 8: 1467-1479.
4. Khonsari F Zakeri-Milani $P$, Jelvehgari M (2014) Formulation and Evaluation of In-vitro Characterization of Gastic-Mucoadhesive Microparticles/Discs Containing Metformin Hydrochloride. Iran J Pharm Res 13: 67-80.

5. Al Kindi H, Paul A, You Z, Nepotchatykh O, Schwertani A, et al. (2014) Sustained release of milrinone delivered via microparticles in a rodent model of myocardial infarction. J Thorac Cardiovasc Surg 148: 2316-2323. 